\section{LESSON 18}

MARGINS:

Left, Pica 20; Elite 30;

Right, moved out of the way.

PAPER: Against the paper guide at 0 on the scale on the left. Turn up 7 single lines.

Check the 'ready-to-type' position: feet, body, fingers and eyes.

\section{Warm up}

Although two spaces are suggested in this book, one or three spaces are allowed by certain examiners. Check to see what is allowed in your examining area.

Revise numbers

Keep your eyes on the copy.

Figure 2

Practise the reach until you can type 2 with the $s$ finger without looking down. Keep the f finger in position.

\section{Figure 9}

Practise the reach until you can type 9 with the I finger without looking down. Keep the $j$ finger in position.

\section{Roman numerals}

While the numerals can be either capital or small letters, see that they are not mixed.

See that the paper is taken out of the machine correctly and the machine put away in the approved manner.

UNIT 5

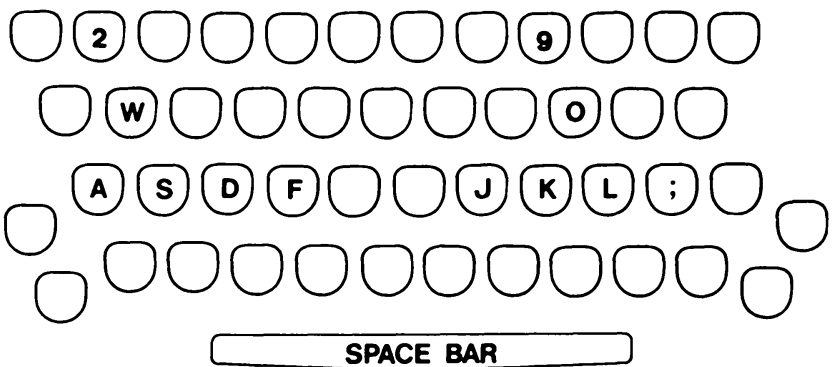

Remember to leave a blank line between sections.

Type these two paragraphs exactly as they are as many times as your teacher asks. In order to type the lines after the first line in each paragraph you will have to tap twice on the space bar first.

A Hanging paragraph is one in which the first line starts two spaces to the left of the other lines in the paragraph.

These two paragraphs are in the Hanging style. If you are ever asked to type in the Hanging style, this is it.

Type each line 3 times. Tap the keys sharply and try to build up speed.

1. If 34 more come, there will be 156 here.

2. In 47 games there were 184 goals scored.

3. Over 85 books were written by the 7 men.

4. In 7 hours 475 people came to the house.

Type the first line of each new figure as many times as your teacher asks.

Type the other lines indicated by your teacher 3 times each.

5. sss sws sw2 sw2 sw2f s $2 f$ f $2 s$ s $2 s$ s $2 f$ s $2 s$

6. 2 times 2 is 4 but 2 minus 2 is nothing.

7. If 2 times 2 times 2 makes 8 it is done.

8. 111 lol $109109191 \quad 19 j \quad 19 j 919 j 191191$

9. 9 and 9 and $O$ less 9 and 9 and $O$ is nil.

10. When the 9 boys have hit 99 we have won.

Sometimes, roman numerals are used instead of our arabic notation. Letters are used for numerals and either capital or small letters are used. Use a capital I for one, $V$ for five and $X$ for ten. If you wish, of course, you can use the small $i$, the small $v$ and the small $x$. In Lesson 126 you will be told more about roman numerals.

Type this paragrapn as many times as your teacher asks. Type it using both capital and small letters for the numerals.

11. One is $I$, five is $V$ and ten is $X$. Three is III, two is II, four is IV and six is VI while seven is VII and eight is VIII. Type a one before ten, IX, to make nine.

\section{LESSON 18}

